\title{
Viscosities of Quark-Gluon Plasmas
}

\author{
H. Heiselberg \\ Nuclear Science Div., MS 70A-3307, Lawrence Berkeley Laboratory, Berkeley, CA 94720, USA
}

The quark and gluon viscosities are calculated in quark-gluon plasmas to leading orders in the coupling constant by including screening. For weakly interaction QCD and QED plasmas dynamical screening of transverse interactions and Debye screening of longitudinal interactions controls the infrared divergences. For strongly interacting plasmas other screening mechanisms taken from lattice calculations are employed. By solving the Boltzmann equation for quarks and gluons including screening the viscosity is calculated to leading orders in the coupling constant. The leading logarithmic order is calculated exactly by a full variational treatment. The next to leading orders are found to be very important for sizable coupling constants as those relevant for the transport properties relevant for quark-gluon plasmas created in relativistic heavy ion collisions and the early universe. 


\section{INTRODUCTION}

Transport and relaxation properties of quark and gluon (QCD) plasmas are important in a number of a different contexts. They determine the time that it takes a quarkgluon plasma formed in a heavy-ion collision to approach equilibrium, and they are of interest in astrophysical situations such as the early universe, and possibly neutron stars.

The basic difficulty in calculating transport properties of such plasmas, as well as of relativistic electron-photon (QED) plasmas, is the singular nature of the long-range interactions between constituents, which leads to divergences in scattering cross sections similar to those for Rutherford scattering. This makes the problem of fundamental methodological interest, in addition to its possible applications. The first approaches to describe the transport properties of quark-gluon plasmas employed the relaxation time approximation [1, 2, 3] for the collision term. This approximation simplifies the collision integral enormously and transport coefficients are related directly to the relaxation time. The latter is typically estimated from a characteristic cross section times the density of scatterers. In Refs. [2, 3] the divergent part of the total cross section at small momentum transfers was assumed to be screened at momentum transfers less than the Debye momentum. However, Debye screening influences only the longitudinal (electric) part of the QED and QCD interactions, and the transverse (magnetic) part is unscreened in the static limit.

Recently it has been shown that the physics responsible for cutting off transverse interactions at small momenta is dynamical screening [4]. This effect is due to Landau damping of the exchanged gluons or photons. Within perturbative QCD and QED rigorous analytical calculations of transport coefficients to leading order have been made for temperatures high [4,5] as well as low [6] compared with the chemical potentials of the constituents.

Transport processes depend on a characteristic relaxation time, $\tau_{t r}$, of the particular transport process considered. For example, in high temperature plasma the viscosities, $\eta_{i}=w_{i} \tau_{\eta, i} / 5$, of particle type $i$ are proportional to the characteristic times for viscous relaxation, $\tau_{\eta, i} \sim \tau_{t r}$, which were first calculated in [4] to leading order in the coupling constant. More generally one finds that the typical transport relaxation rates, that determines momentum stopping, thermal and viscous relaxation, is in a weakly interacting QCD plasma

$$
\frac{1}{\tau_{t r}} \propto \alpha_{s}^{2} \ln \left(1 / \alpha_{s}\right) T+O\left(\alpha_{s}^{2}\right)
$$

where the expansion is in terms of the fine structure constant $\alpha_{s}=g^{2} / 4 \pi$. The coefficients of proportionality to the leading order in $\alpha_{s}$ (in the following called the leading logarithmic order) has been calculated analytically for a number of transport processes in high temperature plasmas [4. Likewise in a QED plasma the typical transport relaxation rates for viscous processes, mome tum stopping, thermal and electrical conduction have $t$ same dependence as (11) on the QED fine structure cc stant $\alpha[5]$.

The dependence of the transport rates on the coupli constants is very sensitive to the screening. Besides $t$ factor $\alpha_{s}^{2}$ from the matrix element squared of the qua and gluon interactions, the very singular QCD inter tions for small momentum transfers lead to a logarith $\ln \left(q_{\max } / q_{\min }\right)$, of the maximum and minimum mome tum transfers. The typical particle momenta limits $t$ maximum momentum transfer, $q_{\max } \sim T$, and Deb and dynamical screening leads to effective screening small momentum transfers of order $q_{\text {min }} \sim q_{D} \sim g$ This gives the leading logarithmic order in the coupli constant, $\ln \left(T / q_{D}\right) \sim \ln \left(1 / \alpha_{s}\right)$, to the transport ra (11).

The calculations in [4,5] were brief and dealt only w the leading logarithmic order in the coupling consta with a given ansatz for the distribution function. He more detailed calculations of the quark and gluon $v$ cosities in the high temperature quark-gluon plasmas c presented. The leading logarithmic order is calculat exactly by a variational method and the next to leadi order - the $\alpha_{s}^{2}$ term in (11) - is calculated as well. Becal $\alpha_{s}$ is not exponentially small, the next to leading orc is important in many realistic physical situations as $r$ ativistic heavy ion collisions and the early universe. F thermore when the Debye screening length is larger th the interparticle screening, which occur when $\alpha_{s} \gtrsim 0.1$ we shall see below, Debye and dynamical screening brea down. Instead lattice gauge calculations have found th quark-gluon plasmas seem to develop a constant scree ing mass, $m_{p l} \simeq 1.1 T$, for temperatures $T \gtrsim 2-3 T_{c}$ and is important to see what effects this alternative screeni mechanism has in strongly interacting plasmas.

We shall first describe in section II the transport thec we use, namely the Boltzmann equation, and the scree ing of long range QCD and QED interactions. In secti III, we describe the process of shear flow and the var tional calculation necessary in order to find the viscosi In section IV we then evaluate the collision term to lea ing logarithmic order with a simplifying ansatz for $t$ trial function and refer to Appendix A for a full and act variational calculation. In section $\mathrm{V}$ we calculate $\mathrm{t}$ viscosity to higher orders in the coupling constant a discuss strongly interacting plasmas. Finally, in secti VI we give a summary and discuss generalizations of $t$ methods developed here to other transport coefficient

\section{TRANSPORT THEORY}

Transport processes are most easily described by $t$ Boltzmann equation

$$
\left(\frac{\partial}{\partial t}+\mathbf{v}_{1} \cdot \nabla_{\mathbf{r}}+\mathbf{F} \cdot \nabla_{\mathbf{p}_{1}}\right) n_{1}=2 \pi \nu_{2} \sum_{234}|M|^{2}
$$




$$
\begin{aligned}
& \times\left[n_{1} n_{2}\left(1 \pm n_{3}\right)\left(1 \pm n_{4}\right)-\left(1 \pm n_{1}\right)\left(1 \pm n_{2}\right) n_{3} n_{4}\right] \\
& \times \delta\left(\epsilon_{1}+\epsilon_{2}-\epsilon_{3}-\epsilon_{4}\right) \delta_{\mathbf{p}_{1}+\mathbf{p}_{2} ; \mathbf{p}_{3}+\mathbf{p}_{4}}
\end{aligned}
$$

where $\epsilon_{i}$ is the energy and $\mathbf{p}_{i}$ the momentum of the quasiparticles, $\mathbf{F}$ some force acting on the quasi-particles, and the right hand side of (2) is the collision term. $n_{i}\left(\mathbf{p}_{i}\right)$ are the Fermi and Bose quasi-particle distribution functions for quarks and gluons and the signs \pm include stimulated emission and Pauli blocking. The spin and color statistical factor $\nu_{2}$ is 16 for gluons and $12 N_{f}$ for quarks and antiquarks with $N_{f}$ flavors. $|M|^{2}$ is the squared matrix element for the scattering process $12 \rightarrow 34$, summed over final states and averaged over initial states. It is related to the Lorentz-invariant matrix element $|\mathcal{M}|^{2}$ by $|M|^{2}=|\mathcal{M}|^{2} /\left(16 \epsilon_{1} \epsilon_{2} \epsilon_{3} \epsilon_{4}\right)$. For gluon-gluon scattering [7] (see Fig. (11)

$$
\left|\mathcal{M}_{g g}\right|^{2}=\frac{9}{4} g^{4}\left(3-\frac{u s}{t^{2}}-\frac{s t}{u^{2}}-\frac{u t}{s^{2}}\right),
$$

where $s, t$, and $u$ are the usual Mandelstam variables. In Eq. (3) the double counting of final states has been corrected for by inserting a factor $1 / 2$. For quark-gluon scattering

$$
\left|\mathcal{M}_{g q}\right|^{2}=g^{4}\left(u^{2}+s^{2}\right)\left(\frac{1}{t^{2}}-\frac{4}{9 u s}\right),
$$

and for scattering of two different quark flavors

$$
\left|\mathcal{M}_{q_{1} q_{2}}\right|^{2}=\frac{4}{9} g^{4} \frac{u^{2}+s^{2}}{t^{2}} .
$$

The matrix element for scattering of the same quark flavors or quark-antiquark scattering is different at large momentum transfer but the same as (5) at small momentum transfers.

The $t^{-2}$ and $u^{-2}$ singularities in Eq. (35) lead to diverging transport cross sections and therefore vanishing transport coefficients. Including screening, it was shown in [4,5.6] that finite transport coefficients are obtained. In fact, the leading contribution to transport coefficients comes from these singularities. In the $t=\omega^{2}-q^{2}$ channel the singularity occurs for small momentum $\mathbf{q}$ and energy $\omega$ transfers (see Fig. (11)).

For small momentum transfer, $q \ll \epsilon_{1}, \epsilon_{2} \sim T$, energy conservation implies that $\omega=\epsilon_{1}-\epsilon_{3} \simeq \mathbf{v}_{1} \cdot \mathbf{q}=-\mathbf{v}_{2} \cdot \mathbf{q}$ where $\mathbf{v}_{i}=\mathbf{p}_{i}$. Therefore the velocity projections transverse to $\mathbf{q}$ have lengths $\left|\mathbf{v}_{1, T}\right|=\left|\mathbf{v}_{2, T}\right|=\sqrt{1-\mu^{2}}$, where $\mu=\omega / q$. Consequently $\mathbf{v}_{1, T} \cdot \mathbf{v}_{2, T}=\left(1-\mu^{2}\right) \cos \phi$, where $\phi$ is the angle between $\mathbf{v}_{1, T}$ and $\mathbf{v}_{2, T}$. For $q \ll T$ we thus have

$$
\begin{aligned}
s \simeq-u & \simeq 2 p_{1} p_{2}\left(1-\cos \theta_{12}\right) \\
& \simeq 2 p_{1} p_{2}(1-\mu)(1-\cos \phi)
\end{aligned}
$$

and the interactions splits into longitudinal and transverse ones, \&

$$
\left|M_{g g}\right|^{2}=\frac{9}{8} g^{4}\left|\frac{1}{q^{2}+\Pi_{L}}-\frac{\left(1-\mu^{2}\right) \cos \phi}{q^{2}-\omega^{2}+\Pi_{T}}\right|^{2} .
$$

The interactions are modified by inclusion of the gluc or photon, self energies, $\Pi_{L}$ and $\Pi_{T}$ [8] (see also Fig. ( In the random-phase approximation the polarizations : given in the long wavelength limit $(q \ll T)$ by

$$
\begin{aligned}
& \Pi_{L}(q, \omega)=q_{\mathrm{D}}^{2}\left[1-\frac{\mu}{2} \ln \left(\frac{\mu+1}{\mu-1}\right)\right], \\
& \Pi_{T}(q, \omega)=q_{\mathrm{D}}^{2}\left[\frac{\mu^{2}}{2}+\frac{\mu\left(1-\mu^{2}\right)}{4} \ln \left(\frac{\mu+1}{\mu-1}\right)\right],
\end{aligned}
$$

where $\mu=\omega / q$ and $q_{\mathrm{D}}=1 / \lambda_{\mathrm{D}}$ is the Debye wavenumb In a weakly-interacting high temperature QCD plasn 8.9.9.

$$
q_{D}^{2}=4 \pi\left(1+N_{f} / 6\right) \alpha_{s} T^{2},
$$

where $\alpha_{s}=g^{2} / 4 \pi$ is the fine structure constant for stro interactions, the factor $\left(1+N_{f} / 6\right)$ is the sum of contrib tions from gluon screening, the " 1 ," and from light me quarks, of number of flavors, $N_{f}$. In a high temperat QED plasma, $q_{\mathrm{D}}^{2}=4 \pi \alpha T^{2} / 3$, where $\alpha$ is the QED fi structure constant.

One should keep in mind that the self energies of and (9) are only valid in the long wavelength limit, i. for $q \ll T$. When $q \sim T$ other contributions of orc $\alpha_{s} q T$ enter (see, e.g., [8]) which may be gauge depende 14. However, as long as $\alpha_{s}$ is small all contributic from the self energies can be ignored in the gluon pro agator when $q \sim T$ because the matrix element squar already carry the order $\alpha_{s}^{2}$.

In the above derivations we have consistently assum that the screening was provided in RPA by the glu self energies which give the Debye and dynamical scree ing of longitudinal and transverse interactions resp tively. Both effects provide a natural effective cutoff momentum transfer less than $q_{\text {min }} \sim q_{D}$. These pert bative ideas must, however, break down when the scree ing length becomes as short as the interparticle sp ing, i.e. $q_{D} \sim T$, or in terms of the coupling consta $\alpha_{s} \lesssim\left(4 \pi\left(1+N_{f} / 6\right)\right)^{-1} \sim 0.1$ according to Eq. (10). In 1 tice gauge calculations of quark-gluon plasma above tw the temperature of the phase transition, $T_{c} \simeq 180 \mathrm{Me}$ one finds strong nonperturbative effects in the plasma that the typical screening mass is $m_{p l} \sim 1.1 T[12]$. O may argue 13 that perturbation theory still applies large momentum transfers so that the matrix elemen are given by the simple Feynman tree diagrams, but th perturbation theory does not apply for small momentu transfers of order $q \lesssim q_{D}$ and that one should rather ins the effective cutoff found by lattice gauge calculations

$$
\Pi_{L} \simeq \Pi_{T} \simeq m_{p l}, \quad \alpha_{s} \gtrsim 0.1 .
$$

The phenomenological screening mass of (11) provides with a method to extend our calculations of transport efficients to larger values for $\alpha_{s}$ and it can be combined the Debye and dynamical screening in weakly interacti quark-gluon plasmas. 


\section{THE VISCOSITY}

With screening included in the interaction we can now proceed to calculate transport properties as the viscosity. In the presence of a small shear flow, $\mathbf{u}(y)$, in the $x$ direction we obtain from the Boltzmann equation

$$
\begin{aligned}
& p_{1 x} v_{1 y} \frac{\partial n_{1}}{\partial \epsilon_{1}} \frac{\partial u_{x}}{\partial y}=2 \pi \nu_{2} \sum_{234}|M|^{2} \\
& \times\left[n_{1} n_{2}\left(1 \pm n_{3}\right)\left(1 \pm n_{4}\right)-\left(1 \pm n_{1}\right)\left(1 \pm n_{2}\right) n_{3} n_{4}\right] \\
& \times \delta\left(\epsilon_{1}+\epsilon_{2}-\epsilon_{3}-\epsilon_{4}\right) \delta_{\mathbf{p}_{1}+\mathbf{p}_{2} ; \mathbf{p}_{3}+\mathbf{p}_{4}} .
\end{aligned}
$$

For small $\mathbf{u}$ we can furthermore linearize the quasiparticle distribution function

$$
n_{i}=n_{i}^{L E}+\frac{\partial n}{\partial \epsilon_{p}} \Phi_{i} \frac{\partial u_{x}}{\partial y},
$$

where the local equilibrium distribution function is

$$
n_{i}^{L E}=\left(\exp \left[\left(\epsilon_{i}-\mathbf{u} \cdot \mathbf{p}_{i}\right) / T\right] \mp 1\right)^{-1},
$$

and $\Phi_{i}$ is an unknown function that represents the deviations from local equilibrium. By symmetry $\Phi$ has to be on the form

$$
\Phi=\hat{p}_{x} \hat{p}_{y} f(p / T),
$$

where now the function $f$ must be determined from the Boltzmann equation. Inserting (13) in the Boltzmann equation we find

$$
\begin{aligned}
p_{1 x} v_{1 y} \frac{\partial n_{1}}{\partial \epsilon_{1}} & =2 \pi \nu_{2} \sum_{234}|M|^{2}\left[n_{1} n_{2}\left(1 \pm n_{3}\right)\left(1 \pm n_{4}\right)\right. \\
& \left.-\left(1 \pm n_{1}\right)\left(1 \pm n_{2}\right) n_{3} n_{4}\right] \\
& \times \delta\left(\epsilon_{1}+\epsilon_{2}-\epsilon_{3}-\epsilon_{4}\right) \delta_{\mathbf{p}_{1}+\mathbf{p}_{2} ; \mathbf{p}_{3}+\mathbf{p}_{4}} \\
& \times\left(\Phi_{1}+\Phi_{2}-\Phi_{3}-\Phi_{4}\right) .
\end{aligned}
$$

It is very convenient to define a scalar product of two real functions by:

$$
\left\langle\psi_{1} \mid \psi_{2}\right\rangle=-\nu_{2} \sum_{\mathbf{p}} \psi_{1}(\mathbf{p}) \psi_{2}(\mathbf{p}) \frac{\partial n}{\partial \epsilon_{p}} .
$$

Thus Eq. (16 may be written on the form $|X\rangle=I|\Phi\rangle$ where $|X\rangle=p_{x} v_{y}$ and $I$ is the integral operator acting on $\Phi$. The viscosity is given in terms of $\Phi$ [10] and can now be written as

$$
\eta=-\nu_{2} \sum_{\mathbf{p}} p_{x} v_{y} \frac{\partial n}{\partial \epsilon_{p}} \Phi_{\mathbf{p}}=\langle X \mid \Phi\rangle .
$$

Equivalently, the viscosity is given from (16 as

$$
\eta=\frac{\langle X \mid \Phi\rangle^{2}}{\langle\Phi|I| \Phi\rangle}
$$

Since $\langle. \mid$.$\rangle defines an inner product, the quantity$ $\langle X \mid \Psi\rangle^{2} /\langle\Psi|I| \Psi\rangle$ is minimal for $\Psi=\Phi$ with the minimal value $\eta$. Equation 19 is therefore convenient for var tional treatment, which will be carried out in Appenc A.

To find the viscosity we must solve the integral equ tion (16 for which we have to evaluate

$$
\begin{gathered}
\langle\Phi|I| \Phi\rangle=2 \pi \nu_{2} \sum_{\mathbf{p}_{1}, \mathbf{p}_{2}, \mathbf{q}}|M|^{2} n_{1} n_{2}\left(1 \pm n_{3}\right)\left(1 \pm n_{4}\right) \\
\times \frac{\left(\Phi_{1}+\Phi_{2}-\Phi_{3}-\Phi_{4}\right)^{2}}{4} \delta\left(\epsilon_{1}+\epsilon_{2}-\epsilon_{3}-\epsilon_{4}\right) .
\end{gathered}
$$

Momentum conservation requires that $\mathbf{p}_{3}=\mathbf{p}_{1}+\mathbf{q}$ a $\mathbf{p}_{4}=\mathbf{p}_{2}-\mathbf{q}$ where $\mathbf{q}$ is the momentum transfer. Int ducing an auxilliary integral over energy transfers, $\omega, t$ delta-function in energy can be written

$$
\begin{aligned}
\delta\left(\epsilon_{1}+\epsilon_{2}-\epsilon_{3}-\epsilon_{4}\right) & =\int d \omega \frac{p_{3}}{p_{1} q} \delta\left(\cos \theta_{1}-\mu-\frac{t}{2 p_{1} q}\right) \\
& \times \frac{p_{4}}{p_{2} q} \delta\left(\cos \theta_{2}-\mu+\frac{t}{2 p_{2} q}\right)
\end{aligned}
$$

where $\theta_{1}$ is the polar angle between $\mathbf{q}$ and $\mathbf{p}_{1}$ and $\theta_{2}$ the corresponding one between $\mathbf{q}$ and $\mathbf{p}_{2}$ (see Fig. Consequently, we find

$$
\begin{aligned}
\langle\Phi|I| \Phi\rangle & =\frac{1}{4 \pi^{8} T} \int_{0}^{\infty} d q \int_{-q}^{q} d \omega \\
& \times \int_{(q-\omega) / 2}^{\infty} d p_{1} p_{1}^{2} n_{1}\left(p_{1}\right)\left(1 \pm n_{1}\left(p_{1}+\omega\right)\right) \\
& \times \int_{(q+\omega) / 2}^{\infty} d p_{2} p_{2}^{2} n_{2}\left(p_{2}\right)\left(1 \pm n_{2}\left(p_{2}-\omega\right)\right) \\
& \times \int_{0}^{2 \pi} \frac{d \phi}{2 \pi}|M|^{2}\left(\Phi_{1}+\Phi_{2}-\Phi_{3}-\Phi_{4}\right)^{2}
\end{aligned}
$$

This integral equation for $\Phi$ has been solved in a $f$ cases under simplifying circumstances. F.ex., in Fer liquids the sharp Fermi surface restricts all particle $\mathrm{m}$ menta near the Fermi surface and with a simplified for for the scattering matrix element techniques have be developed to calculate a number of transport coefficies exactly [10]. For the QCD and QED plasmas the very s gular interaction can, once screened, be exploited since allows an expansion at small momentum transfers. Th an analytical calculation of the transport coefficients c be carried out at least to leading logarithmic order in t coupling constant [4,5, 6].

\section{VISCOSITY TO LEADING LOGARITHMIC ORDER}

In Ref. [4], through solution of the Boltzmann kine equation, the first viscosity of a quark-gluon plasma n derived to leading logarithmic order in the QCD coupli strength. We will in the following give a more thorou and exact derivation of the quark and gluon viscosi 
The total viscosity, to leading order, is an additive sum of the gluon and quark viscosities, $\eta=\eta_{g}+\eta_{q}$.

The leading logarithmic order comes from small momentum transfers because of the very singular matrix element (7) dominates. For small $q$ the kinematics simplify enormously and, as we will now show, the integrals separate allowing almost analytical calculations. First, we can set the lower limits on the $p_{1}$ and $p_{2}$ integrals to zero, however, then replacing the upper limit on $q$ by the natural cutoff from the distribution functions which is $q_{\max } \sim T$. Thus we find from (22)

$$
\begin{aligned}
\langle\Phi|I| \Phi\rangle & =\frac{1}{2 \pi^{8} T} \int_{0}^{\infty} d p_{1} p_{1}^{2} n_{1}\left(1 \pm n_{1}\right) \\
& \times \int_{0}^{\infty} d p_{2} p_{2}^{2} n_{2}\left(1 \pm n_{2}\right) \\
& \times \int_{0}^{q_{\max }} q d q \int_{-1}^{1} \frac{d \mu}{2} \int_{0}^{2 \pi} \frac{d \phi}{2 \pi} \\
& \times|M|^{2}\left(\Phi_{1}+\Phi_{2}-\Phi_{3}-\Phi_{4}\right)^{2} .
\end{aligned}
$$

to leading logarithmic order

The solution to the integral equation or equivalently the variational calculation of $(19)$ is quite technical and is for that reason given in Appendix A. A much simpler calculation is to make the standard assumption in viscous processes, i.e., to take the trial function as

$$
f(p / T)=(p / T)^{2} .
$$

As will be shown in Appendix A this turns out to be a very good approximation. It is accurate to more than $99 \%$ for reasons also explained in the appendix. $f$ can be defined up to any constant which cancels in (19) and therefore never enters in the viscosity.

The quantity $\left(\Phi_{1}+\Phi_{2}-\Phi_{3}-\Phi_{4}\right)^{2}$ can be averaged over $x$ - and $y$-directions while keeping $\mu$ and $\phi$ fixed. This corresponds to keeping the relative positions of the three vectors $\mathbf{q}, \mathbf{p}$, and $\mathbf{p}^{\prime}$ fixed relative to each other and rotating this system over the three Euler angles (see also Appendix A). Consequently, we obtain

$$
\begin{aligned}
&\left\langle\left(\Phi_{1}+\Phi_{2}-\Phi_{3}-\Phi_{4}\right)^{2}\right\rangle= \frac{q^{2}}{15 T^{4}}\left[3\left(\mathbf{p}_{2}-\mathbf{p}_{1}\right)^{2}\right. \\
&\left.+\left(\hat{\mathbf{q}} \cdot\left(\mathbf{p}_{2}-\mathbf{p}_{1}\right)\right)^{2}\right] \\
&=\frac{q^{2}}{15 T^{4}}\left[\left(3+\mu^{2}\right)\left(p_{1}^{2}+p_{2}^{2}\right)\right. \\
&\left.\quad-2 p_{1} p_{2}\left(4 \mu^{2}+3\left(1-\mu^{2}\right) \cos \phi\right)\right] .
\end{aligned}
$$

The integrals over $p_{1}$ and $p_{2}$ in (23) are elementary. Next we perform the integrations or averages over $\mu$ and $\phi$ required in (23). We note in passing that the term in (25) proportional to $p_{1} p_{2}$ vanishes and that $\mu^{2}$ effectively can be replaced by $1 / 3$ (see Appendix A). Let us first consider the case of gluon-gluon scattering inserting $\left|M_{g g}\right|^{2}$ from (3). We thus find

$$
\begin{aligned}
\langle\Phi|I| \Phi\rangle= & \frac{2^{8} \pi^{3}}{45} \alpha_{s}^{2} T^{3} \int_{0}^{q_{\max }} q^{3} d q \int_{0}^{1} d \mu \\
& {\left[\frac{1}{\left|q^{2}+\Pi_{L}(\mu)\right|^{2}}+\frac{1 / 2}{\left|q^{2}+\Pi_{T}(\mu) /\left(1-\mu^{2}\right)\right|^{2}}\right.}
\end{aligned}
$$

This integral is discussed in detail in appendix B. $\mathrm{F}$ the longitudinal interactions $\Pi_{L} \simeq q_{D}^{2}$ due to Deb screening and the leading term is a logarithm of $t$ ratio of maximum to minimum momentum transf $\ln \left(q_{\text {max }} / q_{\text {min }}\right) \sim \ln \left(T / q_{D}\right)$. Likewise for the transve interactions $\Pi_{T} \simeq i(\pi / 4) \mu q_{D}^{2}$ due to Landau dam ing and the dependence on $\mu=\omega / q$ provides sufficie screening to render the integral finite and the leadi term is the same logarithm as for the longitudinal int actions. Whereas the details of the screening are uni portant for the leading logarithmic order, they are $\mathrm{i}$ portant for the higher orders and they are calculated detail in appendix B. The final result is thus to leadi logarithmic order

$$
\langle\Phi|I| \Phi\rangle=\frac{2^{7} \pi^{3}}{15} \alpha_{s}^{2} \ln \left(T / q_{D}\right) T^{3},
$$

Since $\langle\Phi \mid X\rangle=\left(64 \xi(5) / \pi^{2}\right) T^{3}$, we find from (19) and $(2$

$$
\begin{aligned}
\eta_{g g} & =\frac{2^{5} 15 \xi(5)^{2}}{\pi^{7}} \frac{T^{3}}{\alpha_{s}^{2} \ln \left(T / q_{D}\right)} \\
& \simeq 0.342 \frac{T^{3}}{\alpha_{s}^{2} \ln \left(1 / \alpha_{s}\right)},
\end{aligned}
$$

to leading logarithmic order in $\alpha_{s}=g^{2} / 4 \pi$.

To obtain the full gluon viscosity we must add scatt ing on quarks and antiquarks which is calculated ana gously and only has a few factors different. Firstly, frc (3) and (4) we see that the matrix element squared a factor $4 / 9$ smaller. Secondly, the statistical factor $\nu_{2}=12 N_{f}$ instead of 16 . Thirdly, in integrating or the factor $\left(p_{1}^{2}+p_{2}^{2}\right)$ in Eq. 25) we note that the dist bution function, $n_{2}$, in Eq. (23) is now a fermion or Consequently, the $p_{1}$ and $p_{2}$ integrations give a fact $(1 / 2+7 / 8) / 2$ less for gluon-quark collisions as compar to gluon-gluon collisions and we find

$$
\eta_{g}=\left(\eta_{g g}^{-1}+\eta_{g q}^{-1}\right)^{-1}=\frac{\eta_{g g}}{1+11 N_{f} / 48} .
$$

In 泪 the slightly different result $\eta_{g}=\eta_{g g} /\left(1+N_{f}\right)$ was obtained.

The quark viscosity can be obtained analogously the gluon one. The quark viscosity due to collisions quarks only, $\eta_{q q}$, deviates from $\eta_{g g}$ by a factor $(4 / 9)^{2}$ the matrix elements and differences in having Fermi a Bose integrals. By comparing to (19, 18,20) we find

$$
\eta_{q q}=\eta_{g g} \frac{(15 / 16)^{2}}{(4 / 9)^{2}(7 / 8)(1 / 2)}=\eta_{g g} \frac{5^{2} 3^{6}}{2^{8} 7} .
$$


Note that the statistical factors $\nu$ cancel in $\eta_{g g}$ and $\eta_{q q}$. Including quark scatterings on gluons lead to similar factors in in $\langle\Phi|I| \Phi\rangle$, namely a factor $(9 / 4)$ from the matrix element, a factor $16 / 12 N_{f}$ from statistics, and a factor $(8 / 7+2) / 2$ from Bose instead of Fermi integrals. Thus

$$
\eta_{q}=\frac{\eta_{q q}}{1+33 / 7 N_{f}} \simeq 2.2 \frac{1+11 N_{f} / 48}{1+7 N_{f} / 33} N_{f} \eta_{g},
$$

which for $N_{q}=2$ results in $\eta_{q}=4.4 \eta_{g}$, a quark viscosity that is larger than the gluon one partly because the gluons generally interact stronger than the quarks and partly because of differences between Bose and Fermi distribution functions.

\section{VISCOSITY TO HIGHER ORDERS IN $\alpha_{S}$}

The leading logarithmic order dominates at extremely high temperatures, where the running coupling constant is small, but it is insufficient at lower temperatures. The next to leading order correction to the viscous rate in the coupling constant is of order $\alpha_{s}^{2}$. It may be significant because the leading logarithm is a slowly increasing function. In the derivation of the leading logarithmic order, Eq. (27), we have been very cavalier with any factors entering in the logarithm, which are of order $\alpha_{s}^{2}$. It was only argued that the leading logarithmic order $\ln \left(q_{\max } / q_{\min }\right) \sim \ln \left(T / q_{D}\right)$ because $q_{\max }$ and $q_{\min }$ were of order $\sim T$ and $\sim q_{D}$ respectively. Finally, if thermal quark-gluon plasmas are created in relativistic heavy ion collisions at CERN and RHIC energies, the temperatures achieved will probably be below a $\mathrm{GeV}$. We can thus estimate the interaction strength from the running coupling constant $\alpha_{s} \simeq 6 \pi /\left(33-2 N_{f}\right) \ln (T / \Lambda)$ which, with $\Lambda \simeq 150 \mathrm{MeV}$ and $T \lesssim 1 \mathrm{GeV}$, gives $\alpha_{s} \gtrsim 0.4$. For such large coupling constants Debye and dynamical screening is replaced by an effective screening mass, $m_{p l}$, as discussed above which will affect the viscosity considerably.

To calculate the viscosity to order $\alpha_{s}^{2}$ exactly, the 5dimensional integral of (22) must be evaluated numerically and at the same time a variational calculation of $\Phi$ must be performed. This is a very difficult task and we shall instead use the information obtained in the previous section, that the trial function $f \propto p^{2}$ is expected to be an extremely good approximation. With that ansatz for the trial function, it is then straight forward to calculate the integral of (21) numerically and find the viscosity to order $\alpha_{s}^{2}$ for the given screening mechanism. The 5dimensional numerical evaluation of the collision integral of (21) is a complicated function of the coupling constant. It is convenient to write it in terms of the function $Q$

$$
\langle\Phi|I| \Phi\rangle_{g g}=\frac{2^{7} \pi^{3}}{15} \alpha_{s}^{2} Q\left(\frac{q_{\max }^{(g g)}}{q_{\min }}\right) T^{3},
$$

where the index $g g$ refers to gluon-gluon scattering but the analogous definitions applies to gluon-quark and quark-quark scattering. The function $Q$ and the eff tive maximum and minimum momentum transfer, $q_{m}$ and $q_{\text {min }}$, are given in Appendix B. In weakly intera ing plasmas, where the screening is provided by Deb and dynamical screening, the function $\mathrm{Q}$ is basically $\mathrm{j}$ a logarithm of the ratio of the maximum and minim momentum transfer, i.e.,

$$
Q\left(\frac{q_{\max }}{q_{\min }}\right)=\ln \left(\frac{q_{\max }}{q_{\min }}\right), \quad \alpha_{s} \lesssim 0.1
$$

By numerical integration we find that the distributi functions leads to an effective cutoff of $q_{\max }^{(g g)} \sim 3 T$. T is because the distribution functions are weighted wi several powers of particle momenta and thus contribu the most for $p \simeq 3 T$. The effective cutoff is slightly lar for quark-gluon and quark-quark scattering because $t$ Fermi distribution functions emphasize large momer than the Bose ones. Debye and dynamical screening lea to $q_{\text {min }} \simeq 1.26 q_{D}$ as described in Eq. (B6) and so fro (B8)

$$
Q\left(\frac{q_{\max }^{(g g)}}{q_{\min }}\right)=\ln \left(\frac{0.44}{\alpha_{s}\left(1+N_{f} / 6\right)}\right), \quad \alpha_{s} \lesssim 0.1 .
$$

The numerical factor inside the logarithm, which gi the order $\alpha_{s}^{2}$, is discussed in more detail in Appendix

In the other limit, $q_{D} \gtrsim T$ or equivalently $\alpha_{s} \gtrsim 0.1, \mathrm{p}$ turbative ideas breaks down and we assume an effect screening mass taken from lattice calculations, $q_{\min }$ $1.1 T$, as described by Eq. (11). Thus we find (see (B10

$$
Q\left(q_{\max }^{(g g)} / q_{\min }\right)=0.626, \quad \alpha_{s} \gtrsim 0.1,
$$

and similarly for quark-gluon and gluon-gluon scatt ing $Q\left(q_{\max }^{(g q)} / q_{\min }\right)=0.819$ and $Q\left(q_{\max }^{(q q)} / q_{\min }\right)=1.0$ respectively.

Adding gluon-gluon and gluon-quark scatterings obtain the gluon viscosities

$$
\begin{aligned}
\eta_{g}= & \frac{2^{5} 15 \xi(5)^{2}}{\pi^{7}} \frac{T^{3}}{\alpha_{s}^{2}} \\
& {\left[Q\left(\frac{q_{m a x}^{(g g)}}{q_{D}}\right)+\frac{11 N_{f}}{48} Q\left(\frac{q_{m a x}^{(g q)}}{q_{D}}\right)\right]^{-1}, }
\end{aligned}
$$

which extends Eq. (37) to higher orders. In weakly teracting plasmas (36) reduces to

$$
\begin{aligned}
\eta_{g} \simeq & 0.342 \frac{T^{3}}{\alpha_{s}^{2}}\left[\ln \left(\frac{0.44}{\alpha_{s}\left(1+N_{f} / 6\right)}\right)\right. \\
& \left.+\frac{11 N_{f}}{48} \ln \left(\frac{0.72}{\alpha_{s}\left(1+N_{f} / 6\right)}\right)\right]^{-1}, \alpha_{s} \lesssim 0.1
\end{aligned}
$$

to leading orders in $\alpha_{s}$. In strongly interacting plasm we obtain by inserting (B10) in (32)

$$
\eta_{g} \simeq 0.55 \frac{T^{3}}{\alpha_{s}^{2}}\left[1+1.31 \frac{11 N_{f}}{48}\right]^{-1}, \alpha_{s} \gtrsim 0.1
$$


In Fig. (3) we show the gluon viscosity with the various assumptions for screening. With dash-dotted curve the result of Eq. (38) assuming a constant screening mass, $m_{p l}=1.1 T$, is shown. With dashed curve the numerical result assuming Debye and dynamical screening of Eqs. (8) and (9) is shown. For $\alpha_{s} \lesssim 0.05$ it is given by Eq. (37) to a good approximation whereas for $\alpha_{s} \gtrsim 0.05$ the result of Eq. (B4) is better. The final viscosity shown by full curve is obtained by combining the two limits, i.e., applying Debye and dynamical screening in weakly interacting plasmas when $q_{D} \lesssim T$ or equivalently $\alpha_{s} \lesssim 0.1$ but an effective screening mass $m_{p l}=1.1 T$ as given by Eq. (11) when $\alpha_{s} \gtrsim 0.1$. This corresponds to chosing the smallest value of the viscosities as seen in Fig. (3), i.e., the two limits of Eqs. (37) and (38).

Similarly, adding quark-quark and quark-gluon scatterings we find the quark viscosity

$$
\begin{aligned}
\eta_{q}= & \frac{5^{3} 3^{6} \xi(5)^{2}}{2^{3} 11 \pi^{7}} N_{f} \frac{T^{3}}{\alpha_{s}^{2}} \\
& {\left[Q\left(\frac{q_{\text {max }}^{(g q)}}{q_{D}}\right)+\frac{7 N_{f}}{33} Q\left(\frac{q_{\text {max }}^{(q q)}}{q_{D}}\right)\right]^{-1}, }
\end{aligned}
$$

which in weakly interacting plasmas gives

$$
\begin{aligned}
\eta_{q} \simeq 0.752 N_{f} \frac{T^{3}}{\alpha_{s}^{2}}\left[\ln \left(\frac{0.72}{\alpha_{s}\left(1+N_{f} / 6\right)}\right)\right. \\
\left.+\frac{7 N_{f}}{33} \ln \left(\frac{1.15}{\alpha_{s}\left(1+N_{f} / 6\right)}\right)\right]^{-1}, \alpha_{s} \lesssim 0.1,
\end{aligned}
$$

and in the strongly interacting plasmas

$$
\eta_{q} \simeq 0.92 N_{f} \frac{T^{3}}{\alpha_{s}^{2}}\left[1+1.25 \frac{7 N_{f}}{33}\right]^{-1}, \alpha_{s} \gtrsim 0.1 .
$$

The quark viscosity increases with the number of quark flavors, $N_{f}$, whereas the gluon viscosity decreases as can be seen in Fig. (4) and (5), where the viscosities are shown for two and three flavors respectively. The total viscosity of a quark-gluon plasma, $\eta=\eta_{g}+\eta_{q}$, is dominated by the quark viscosity.

From the definition of the viscosity in terms of the collision integral (18) and (20), which only contains positive quantities, it follows trivially that the viscosity is positive as is a physical necessity. The resulting viscosities of Eqs. (36) and (39) are positive quantities whereas the $\alpha_{s} \lesssim 0.1$ expansions of Eqs. (37) and (40) are not when extended to the region $\alpha_{s} \gtrsim 0.5$. This explains the results found in [11], where it was claimed that estimates of the next to leading order $\alpha_{s}^{2}$ could lead to a negative viscosity.

Contributions from vertex corrections should also be considered. In fact for the calculation of the quasiparticle damping rate, $\gamma_{p}$, Braaten and Pisarski [15] found that vertex corrections contributed to leading order $\gamma_{p=0}^{(g)} \simeq 6.6 \alpha_{s}$ for zero gluon momenta, $p$. Vertex corrections do also contribute to order $\alpha_{s}$ for large quasiparticle momenta, $p \gg g T$, but they can here be ignored since the leading order is $\gamma_{p}^{(g)}=3 \alpha_{s} \ln \left(1 / \alpha_{s}\right)$ as explain in 16. For the viscosity, however, vertex corrections c be ignored since the extra vertices adds a factor $\alpha_{s}^{2}$. Ev though integration over soft momenta may cancel a $f_{c}$ tor $\alpha_{s}$ the result is still of higher order in the coupli constant.

Writing each of the viscosities $\eta_{i}(i=q, g)$ in terms the viscous relaxation time, $\tau_{\eta i}$, as

$$
\eta_{i}=w_{i} \tau_{\eta i} / 5
$$

where $w_{g}=\left(32 \pi^{2} / 45\right) T^{4}$ and $w_{q}=\left(N_{f} 7 \pi^{2} / 15\right) T^{4}$; the gluon and quark enthalpies respectively, we obt: the viscous relaxation rate for gluons

$$
\begin{aligned}
\frac{1}{\tau_{\eta, g}} & =\frac{\pi^{9}}{3^{3} 5^{3} \xi(5)^{2}} \frac{T}{\alpha_{s}^{2}} \\
& \times\left[Q\left(\frac{q_{\max }^{(g g)}}{q_{\min }}\right)+\frac{11 N_{f}}{48} Q\left(\frac{q_{\max }^{(g g)}}{q_{\min }}\right)\right], \quad \alpha_{s}^{2} T,
\end{aligned}
$$

and quarks and antiquarks

$$
\begin{aligned}
\frac{1}{\tau_{\eta, q}} & =\frac{11 \pi^{9} 2^{3} 7}{3^{7} 5^{5} \xi(5)^{2}} \frac{T}{\alpha_{s}^{2}} \\
& \times\left[Q\left(\frac{q_{\text {max }}^{(g q)}}{q_{\min }}\right)+\frac{7 N_{f}}{33} Q\left(\frac{q_{\max }^{(q q)}}{q_{\min }}\right)\right], \quad \alpha_{s}^{2} T .
\end{aligned}
$$

The viscous relaxation times, $\tau_{\eta, g}, \tau_{\eta, q}$ and $\tau_{\eta}=1 /\left(\tau_{\eta, q}^{-}\right.$ $\left.\tau_{\eta, q}^{-1}\right)$ are thus very similar to the corresponding viscosit when divided by a factor of $T^{4}$. The curves on Fi (4) and (5) therefore applies to the viscous relaxati times (times temperature) as well when divided a fact of $\sim 1.4$ and $\sim 0.92 N_{f}$ for gluons and quarks respective according to Eq. (42).

In weakly interacting plasma the viscous rates can approximated by

$$
\begin{aligned}
\frac{1}{\tau_{\eta, g}} \simeq & 4.11 \alpha_{s}^{2}\left[\ln \left(\frac{0.44}{\alpha_{s}\left(1+N_{f} / 6\right)}\right)\right. \\
& \left.+\frac{11 N_{f}}{48} \ln \left(\frac{0.72}{\alpha_{s}\left(1+N_{f} / 6\right)}\right)\right], \quad \alpha_{s} \lesssim 0.1
\end{aligned}
$$

and

$$
\begin{aligned}
\frac{1}{\tau_{\eta, q}} \simeq 1.27 & \alpha_{s}^{2}\left[\ln \left(\frac{0.72}{\alpha_{s}\left(1+N_{f} / 6\right)}\right)\right. \\
+ & \left.\frac{7 N_{f}}{33} \ln \left(\frac{1.15}{\alpha_{s}\left(1+N_{f} / 6\right)}\right)\right], \alpha_{s} \lesssim 0.1,
\end{aligned}
$$

to leading orders in $\alpha_{s}$.

\section{SUMMARY}

By solving the Boltzmann equation for quarks and $g$ ons the viscosities in quark-gluon plasmas were calc lated to leading orders in the coupling constant. Inclusi 
of dynamical screening of transverse interactions, which controls the infrared divergences in QED and QCD, is essential for obtaining finite transport coefficients in the weakly interacting plasmas. The solution of the transport process was extended to strongly interacting plasmas by assuming an effective screening mass of order $m_{p l}=1.1 T$, as found in lattice calculations, when the Debye screening length became larger than the interparticle distance or when $\alpha_{s} \gtrsim 0.1$. The Boltzmann equation was solved exactly to leading logarithmic order numerically but the result only differed by less than a percent from an analytical result obtained by a simple ansatz for the deviation from local equilibrium, $\Phi \propto p_{x} p_{y}$. The next to leading orders was also calculated and found to be very important for the transport properties relevant for quark-gluon plasmas created in relativistic heavy ion collisions and the early universe. For $\alpha_{s} \gtrsim 0.1$ we find $\eta_{i}=C_{i, 1} T^{3} / \alpha_{s}^{2} \ln \left(C_{i, 2} / \alpha_{s}\right)$ whereas for $\alpha_{s} \gtrsim 0.1$ we find $\eta_{i}=C_{i, 3} T^{3} / \alpha_{s}^{2}$ with coefficients $C_{i, j}$ given above.

The viscosity in degenerate plasmas of quarks, i.e., for $T \ll \mu_{q}$ was calculated in [6]. Several differences were found. In the high temperature quark-gluon plasma the chemical potential can be ignored and the transport processes depend on two momentum scales only, namely $T$ and $q_{D} \sim g T$. In degenerate quark matter three momentum scales enter, namely $\mu_{q}, \mathrm{~T}$, and $q_{D} \sim g \mu_{q}$, and the transport process depends considerably on which of $q_{D}$ and $T$ is the larger. In fact for $T \ll q_{D}$ transverse interactions turn out to be dominant in contrast to the high temperature quark-gluon plasma where transverse and longitudinal interactions contribute by similar magnitude. Furthermore, the existence of a relative sharp Fermi surface allows an almost analytical calculation of both the leading (logarithmic) order as well as the next order $\alpha_{s}^{2}$.

The techniques for calculating the viscosities to leading orders in the coupling constants can be applied to other transport coefficients as well. The leading logarithmic orders to momentum stopping, electrical conductivities and thermal dissipation in QCD and QED plasmas have been estimated with simple ansätze for the distribution functions in [5]. Based on the experience with the viscosity studied here, we do not expect the leading logarithmic order for these transport coefficients to decrease by much when a full variational calculation is performed. The next to leading logarithmic order for these transport coefficients can also be estimated with the experience obtained above for the viscosity. Good estimates are obtained if one in (B5) replaces $q_{\max }$ by the average particle momenta entering the collision integral for the relevant transport process and $q_{\text {min }}$ by $\sim q_{D}$.

A few transport coefficients are, however, different. The second viscosity $\zeta$ is zero for a gas of massless relativistic particles [1] and one cannot define a thermal conductivity in a plasma of zero baryon number. One can, however, consider thermal dissipation processes [5] where the leading orders also can be calculated with the above methods. The effective soft cutoff will, however, be differ- ent for thermal dissipation processes as described in because the transport of energy introduce dependen on $\omega$ which also is present in the transverse screenir $\Pi_{T}(\omega / q)$.

All the transport processes discussed above depe only on momentum scales from the typical parti momentum, $q_{\max } \sim T$ down to the Debye screeni wavenumber $q_{\text {min }} \sim q_{D} \sim g T$ which also is the mome tum scale for dynamical screening. There is, howev a shorter momentum scale of order the magnetic ma $m_{\text {mag }} \sim g^{2} T$, at which perturbative ideas of the qual gluon plasma fails [17]. As shown in 16] the quark a gluon quasiparticle decay rates depend on this infrar cutoff, $m_{\text {mag }}$. Furthermore, recent studies [19] find th the color diffusion and conductivity also depend on $t$ cutoff and therefore the rate of color relaxation is a fact $1 / \alpha_{s}$ larger than Eq. (11).

\section{ACKNOWLEDGMENTS}

This work was supported by DOE grant No. AC03-76SF00098, NSF grant No. PHY 89-21025 a the Danish Natural Science Research Council. Disc sions with Gordon Baym and Chris Pethick are gratefu acknowledged.

\section{APPENDIX A: EXACT VARIATIONAL CALCULATION TO LEADING LOGARITHMI ORDER}

In this appendix we solve the Boltzmann equation a find the deviation from local equilibrium, $\Phi$, by a var tional treatment of Eq. (18).

For a general function $\Phi=\hat{\mathbf{p}}_{x} \hat{\mathbf{p}}_{y} f(p / T)$ we have

$$
\begin{aligned}
\Phi_{1}+ & \Phi_{2}-\Phi_{3}-\Phi_{4}=\hat{\mathbf{p}}_{1, x} \hat{\mathbf{p}}_{1, y} f\left(p_{1}\right)+\hat{\mathbf{p}}_{2, x} \hat{\mathbf{p}}_{2, y} f\left(p_{2}\right) \\
& -\hat{\mathbf{p}}_{3, x} \hat{\mathbf{p}}_{3, y} f\left(p_{3}\right)-\hat{\mathbf{p}}_{4, x} \hat{\mathbf{p}}_{4, y} f\left(p_{4}\right) \\
= & -\left(\hat{\mathbf{q}}_{x} \hat{\mathbf{p}}_{y}+\hat{\mathbf{q}}_{y} \hat{\mathbf{p}}_{x}\right) f(p)-\mu \hat{\mathbf{p}}_{x} \hat{\mathbf{p}}_{y} f_{1}(p) \\
& +\left(\hat{\mathbf{q}}_{x} \hat{\mathbf{p}}_{y}^{\prime}+\hat{\mathbf{q}}_{y} \hat{\mathbf{p}}_{x}^{\prime}\right) f\left(p^{\prime}\right)+\mu \hat{\mathbf{p}}_{x}^{\prime} \hat{\mathbf{p}}_{y}^{\prime} f_{1}\left(p^{\prime}\right),
\end{aligned}
$$

where we have changed notation to $\mathbf{p}=\mathbf{p}_{1}+\mathbf{q} / 2$ $\mathbf{p}_{3}-\mathbf{q} / 2$ and $\mathbf{p}^{\prime}=\mathbf{p}_{2}-\mathbf{q} / 2=\mathbf{p}_{4}+\mathbf{q} / 2$. We have us that the energy conserving $\delta$-functions of (21) impl $\hat{\mathbf{p}} \hat{\mathbf{q}}=\hat{\mathbf{p}}^{\prime} \hat{\mathbf{p}}=\mu$. Furthermore, have defined the functio

$$
f_{1}(p)=p^{3} d\left(f / p^{2}\right) / d p=p f^{\prime}-2 f,
$$

that vanishes when $f \propto p^{2}$ which was the case for $t$ ansatz used in section $\mathrm{V}$.

For small momentum transfer the matrix element depends only on energy and momentum transfer and $t$ azimuthal angle $\phi$. The $\delta$-functions of (21) taking care energy conservation fixes the polar angles $\theta_{1}$ and $\theta_{2}$ wi respect to q. Thus all the angular integrals for fixed and $\phi$ reduces to rotating the three vectors $\mathbf{q}, \mathbf{p}$ and 
over all Euler angles keeping them fixed relatively to each other. Only $\left(\Phi_{1}+\Phi_{2}-\Phi_{3}-\Phi_{4}\right)^{2}$ depends on the Euler angles and the integration or averaging over the three Eulerian angles, while keeping the relative positions of the vectors $\mathbf{q}, \mathbf{p}$ and $\mathbf{p}^{\prime}$ fixed, i.e. keeping $\mu$ and $\phi$ fixed, gives

$$
\begin{aligned}
& \overline{\left(\hat{\mathbf{q}}_{x} \hat{\mathbf{p}}_{y}+\hat{\mathbf{q}}_{y} \hat{\mathbf{p}}_{x}\right)^{2}}=\overline{\left(\hat{\mathbf{q}}_{x} \hat{\mathbf{p}}_{y}^{\prime}+\hat{\mathbf{q}}_{y} \hat{\mathbf{p}}_{x}^{\prime}\right)^{2}}=\frac{3+\mu^{2}}{15}, \\
& \overline{\left(\hat{\mathbf{p}}_{x} \hat{\mathbf{p}}_{y}\right)^{2}}=\overline{\left(\hat{\mathbf{p}}_{x}^{\prime} \hat{\mathbf{p}}_{y}^{\prime}\right)^{2}}=\frac{1}{15}, \\
& \overline{\left(\hat{\mathbf{q}}_{x} \hat{\mathbf{p}}_{y}+\hat{\mathbf{q}}_{y} \hat{\mathbf{p}}_{x}\right)\left(\hat{\mathbf{p}}_{x} \hat{\mathbf{p}}_{y} \mu\right)}=\frac{2}{15} \mu^{2}, \\
& \overline{\left(\hat{\mathbf{q}}_{x} \hat{\mathbf{p}}_{y}^{\prime}+\hat{\mathbf{q}}_{y} \hat{\mathbf{p}}_{x}^{\prime}\right)\left(\hat{\mathbf{p}}_{x}^{\prime} \hat{\mathbf{p}}_{y}^{\prime} \mu\right)}=\frac{2}{15} \mu^{2}, \\
& \overline{\left(\hat{\mathbf{q}}_{x} \hat{\mathbf{p}}_{y}+\hat{\mathbf{q}}_{y} \hat{\mathbf{p}}_{x}\right)\left(\hat{\mathbf{q}}_{x} \hat{\mathbf{p}}_{y}^{\prime}+\hat{\mathbf{q}}_{y} \hat{\mathbf{p}}_{x}^{\prime}\right)}=\frac{1}{15}\left(3 \hat{\mathbf{p}} \hat{\mathbf{p}}^{\prime}+\mu^{2}\right), \\
& \overline{\left(\hat{\mathbf{q}}_{x} \hat{\mathbf{p}}_{y}+\hat{\mathbf{q}}_{y} \hat{\mathbf{p}}_{x}\right)\left(\hat{\mathbf{q}}_{y}^{\prime} \hat{\mathbf{p}}_{x}^{\prime}\right)}=\overline{\left(\hat{\mathbf{q}}_{x}^{\prime} \hat{\mathbf{p}}_{y}^{\prime}+\hat{\mathbf{q}}_{y}^{\prime} \hat{\mathbf{p}}_{x}^{\prime}\right)\left(\hat{\mathbf{q}}_{y} \hat{\mathbf{p}}_{x}\right)} \\
& =\frac{\mu^{2}}{15}\left(3 \hat{\mathbf{p}} \hat{\mathbf{p}}^{\prime}-1\right), \\
& \overline{\left(\hat{\mathbf{q}}_{y} \hat{\mathbf{p}}_{x}\right)\left(\hat{\mathbf{p}}_{x}^{\prime} \hat{\mathbf{p}}_{y}^{\prime} \mu^{2}\right)}=\frac{\mu^{2}}{30}\left(3\left(\hat{\mathbf{p}} \hat{\mathbf{p}}^{\prime}\right)^{2}-1\right),
\end{aligned}
$$

where $\hat{\mathbf{p}}=\mathbf{p} / \epsilon_{p}$ and $\hat{\mathbf{p}}^{\prime}=\mathbf{p}^{\prime} / \epsilon_{p}^{\prime}$. Since we assume that the plasma temperature is much larger than any of the particle masses, the particles are relativistic and $\hat{\mathbf{p}}, \hat{\mathbf{p}}^{\prime}$ and $\hat{\mathbf{q}}$ are unit vectors. The vector product of $\hat{\mathbf{p}}$ and $\hat{\mathbf{p}}$ is most useful in terms of $\mu$ and $\phi$ (see Fig. (2)

$$
\hat{\mathbf{p}} \hat{\mathbf{p}}^{\prime}=\mu^{2}+\left(1-\mu^{2}\right) \cos \phi .
$$

Next we integrate over $\mu$ and $\phi$. The $\mu$ integration averages $\mu^{2}$ to $1 / 3$ whereas the $\phi$ integration is weighted by a factor $(1-\cos \phi)^{2}$ from the matrix elements. Thus we find that (A7 A9) vanishes whereby all combinations mixing $p_{1}$ and $p_{2}$ very conveniently disappear. After averaging over both Euler angles and $\mu$ and $\phi$ we obtain

$$
\begin{aligned}
\left\langle\overline{\left(\Phi_{1}+\Phi_{2}-\Phi_{3}-\Phi_{4}\right)^{2}}\right\rangle & =\frac{q^{2}}{p^{2}} \frac{1}{15}\left(10 f^{2}+f_{1}^{2}+4 f f_{1}\right) \\
& =\frac{q^{2}}{p^{2}} \frac{2}{5}\left(f^{2}+\frac{1}{6} p^{2} f^{\prime 2}\right) .
\end{aligned}
$$

Let us first consider the pure gluon plasma for which (23) gives

$$
\begin{aligned}
\langle\Phi|I| \Phi\rangle & =\frac{8 \pi}{15} g^{4} \ln \left(T / q_{D}\right) T^{3} \\
& \times \int_{0}^{\infty}\left[f^{2}+\frac{1}{6} p^{2} f^{\prime 2}\right]\left(-\frac{\partial n}{\partial \epsilon_{p}}\right) d p,
\end{aligned}
$$

where $n=(\exp (p / T)-1)^{-1}$ is the gluon distribution function. Since

$$
\langle\Phi \mid X\rangle=\frac{8}{15 \pi^{2}} \int f p^{3}\left(-\frac{\partial n}{\partial \epsilon_{p}}\right) d p,
$$

we find from (18)

$$
\eta=\frac{15}{8 \pi g^{4}} \frac{T^{3}}{\ln \left(T / q_{D}\right)} \frac{\left(\int_{0}^{\infty} f n^{\prime} x^{3} d x\right)^{2}}{\int_{0}^{\infty}\left(f^{2}+\frac{1}{6} x^{2} f^{\prime 2}\right) n^{\prime} d x},
$$

where $x=p / T$. As mentioned above, the function $f(x)$ determined by minimizing (A14). A functional variati with respect to $f$ results in a second order inhomogenec differential equation for $f$

$$
f^{\prime \prime}+\left(\frac{2}{x}+\frac{n^{\prime \prime}}{n^{\prime}}\right) f^{\prime}-\frac{6}{x^{2}} f=-\tilde{C} x,
$$

where $n^{\prime \prime} / n^{\prime}=-(1+2 n)$. $\tilde{C}$ is an arbitrary constant th by rescaling $f$, can be chosen as $\tilde{C}=2$ for convenienc

For $x \gg 1$ we can approximate $n \simeq 0$ and so we find $t$ solution to (A15), that does not increase exponentia for $x \rightarrow \infty$, to be

$$
f(x)=x^{2}, \quad x \gg 1 .
$$

For $x \ll 1$ we can approximate $n \simeq 1 / x$ and the soluti to $($ A15) that is finite at the origin is

$$
f(x)=x^{3}\left(C-\frac{1}{5} \ln x\right), \quad 0 \leq x \ll 1,
$$

where $C \simeq 0.7$ is a constant that can only be determin by finding the full solution to (A15) and matching it A17). This is done by a numerical Runge-Kutta in gration and the result is shown in Fig. (6). The viscos is now found by inserting $f$ in (A14). The exact value $\eta$ thus obtained is only $0.523 \%$ less than the approxime value, $\eta_{g g}$, of Eq. (38). Since the exact value is a va ational minimum, it has to be smaller than that of (2 is only slightly less because $f \simeq x^{2}$ for large as just the ansatz of (24) and $f$ is mainly sampled over values $x=p / T \gg 1$ because the integrals over $p_{1}$ and $p_{2}$ in have powers $\sim p^{4}$ to $\sim p^{5}$ times $n_{p}\left(1+n_{p}\right)$. In Ref. a variational calculation with trial functions $f(p) \propto$ lead to a minimal viscosity for $\nu=2.104$. This result close to the quadratic power of (A16) but tends sligh towards the asymptotic form of A17) (see also Fig. It has almost the same slope and curvature as the ex: solution around $p=5 T$ (note that the absolute value unimportant since it cancels in the viscosity). The cor sponding viscosity was $0.364 \%$ smaller than that of 2 i.e., in between the exact result and the ansatz $f \propto x$

The above analysis was restricted to a pure gl plasma. As mentioned above the distribution functio are weighted with several powers of momentum and do not find much difference between fermions and boso Therefore the deviation from local equilibrium for quan will not be much different from gluons and we can be cc fident that the ansatz, $\Phi \propto p_{x} p_{y}$, of Eq. (24) will be good approximation for quarks as well accurate witl less than a percent. 


\section{APPENDIX B: SOFT AND HARD CONTRIBUTIONS}

The essential contribution to $\langle\Phi|I| \Phi\rangle$ is the integral

$$
\begin{aligned}
Q\left(\frac{q_{\max }}{q_{\min }}\right)=\frac{1}{3} & \int_{-1}^{1} d \mu \int_{0}^{q_{\max }} q^{3} d q\left[\frac{1}{\left|q^{2}+\Pi_{L}(\mu)\right|^{2}}\right. \\
& \left.+\frac{1 / 2}{\left|q^{2}+\Pi_{T}(\mu) /\left(1-\mu^{2}\right)\right|^{2}}\right]
\end{aligned}
$$

For dimensional reasons the function $Q$ can only depend on the ratio of $q_{\max }$ to the momentum scale, $q_{\min }$, which is provided by the screening. For Debye and dynamical screening $q_{\min } \sim q_{D}$ whereas lattice calculations of strongly interacting plasmas give $q_{\text {min }} \sim m_{p l}=1.1 T$.

As described in connection with screening nonperturbative effects become important when $q_{D} \gtrsim T$ which corresponds to $\alpha_{s} \lesssim 0.1$. We shall treat the two limits separately starting with the weakly interaction plasmas for which the gluon self energies, $\Pi_{L, T}(\mu)$, are given by Eqs. (8) and (9). It is straight forward to calculate $Q \mathrm{nu}-$ merically and the result will be given below, but let us first make a simple analytical estimate. The main contribution to this integral can be obtained by including the leading terms in the self energies (8, 9)

$$
\begin{aligned}
& \Pi_{L}(q, \omega) \simeq q_{D}^{2}, \\
& \Pi_{T}(q, \omega) \simeq i \frac{\pi}{4} \mu q_{D}^{2} .
\end{aligned}
$$

Thus we find for B1

$$
\begin{aligned}
Q\left(\frac{q_{\max }}{q_{\min }}\right)= & \frac{1}{3}\left[\ln \left(1+\frac{q_{\max }^{2}}{q_{D}^{2}}\right)-\frac{q_{\max }^{2}}{q_{D}^{2}+q_{\max }^{2}}\right. \\
& +\frac{1}{4} \ln \left(1+\frac{q_{\max }^{4}}{q_{D}^{4}}\left(\frac{4}{\pi}\right)^{2}\right) \\
& \left.+\frac{2}{\pi} \frac{q_{\max }^{2}}{q_{D}^{2}} \operatorname{Arctg}\left(\frac{\pi}{4} \frac{q_{D}^{2}}{q_{\max }^{2}}\right)\right] .
\end{aligned}
$$

Expanding in the limit $q_{\max } \gg q_{D}$ or equivalently for small $\alpha_{s}$ we obtain the leading orders up to $\alpha_{s}^{2}$ in the coupling constant

$$
Q\left(\frac{q_{\max }}{q_{\min }}\right) \simeq \ln \left(\frac{q_{\max }}{q_{\min }}\right), \quad q_{\max } \gg q_{D},
$$

where

$$
q_{\text {min }}=q_{D} \exp \left\{\frac{1}{6}\left(1-\ln \frac{4}{\pi}\right)\right\} \simeq 1.13 q_{D} .
$$

The two terms in (B5) corresponding to $\ln q_{\max }$ and $\ln q_{\min }$ are often referred to as "hard" and "soft" contributions in the literature [11.

A numerical evaluation of $(\mathbb{B} 1)$ with $\Pi_{L, T}$ given by Eqs. (8) and (9) instead of (B2) and (B3) gives a slightly larger value for the effective minimum momentum transfer

$$
q_{\min }=1.26 q_{D},
$$

because the additional terms in $\Pi_{L, T}$ lead to some ad tional screening besides the Debye screening and Land

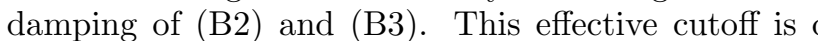
termined by the screening only and is therefore the sa for gluon-gluon, quark-gluon and quark-quark scatteri Whereas $q_{\text {min }}$ may serve as an effective "cutoff" of sm momentum transfers interactions, it is not a parame put in by hand as discussed in 20]. Contrarily, it caused and determined by Debye and dynamical scree ing.

If the transverse interactions are assumed to be Deb screened like the longitudinal ones, i.e., $\Pi_{T}=q_{D}^{2}$, th the result would have been $q_{\min }=q_{D} \exp (0.5)=1.65 q$ This is because dynamical screening of Eq. (B3) is less fective than the Debye screening of (B2) and thus resu in a smaller $q_{\min }$.

It is convenient to express the results in terms of In weakly interacting plasmas we find

$$
\begin{aligned}
Q\left(\frac{q_{\max }}{q_{\min }}\right) & \simeq \ln \left(\frac{q_{\max }}{1.26 q_{D}}\right)= \\
& =\frac{1}{2} \ln \left(\frac{\left(q_{\max } / T\right)^{2}}{4 \pi \alpha_{s}\left(1+N_{f} / 6\right)}\right), \quad \alpha_{s} \lesssim 0.1 .
\end{aligned}
$$

The upper effective cutoff $q_{\max }$ is provided by quark and gluon distribution functions as discussed connection with Eq. (22) and it therefore varies son what with particle type. Because Bose distribution fur tions emphasize smaller momenta than Fermi ones, $q_{m}$ is larger for quarks. We find $q_{\max }^{(g g)}=3.0 T, q_{\max }^{(g q)}=3.8$ and $q_{\max }^{(q q)}=4.8 T$ for gluon-gluon, quark-gluon, a quark-quark scattering respectively. The lower effect cutoff $q_{\min }$ is, however, the same for the three cases cause it only depends on the screening in the gluon pro agator. Furthermore, we find that the extra terms in $t$ matrix elements of (3), (4) and (5) besides the $t^{-2} \mathrm{p}$ do not contribute much since they have varying signs a turn out to be partially cancelling. Thus the constar within the logarithms of (37) and (40) just reflects t different $q_{\max }$ for gluon-gluon, gluon-quark and qua quark scattering.

Lacking screening of transverse interactions in static limit, it has often been assumed that some mec anism like Debye screening might lead to screening transverse interactions as well, i.e. $m_{p l}=q_{D}$. Recent lattice gauge calculations of QCD plasmas have fou effective screening masses of order $m_{p l} \simeq 1.1 T$ near t phase transition point, $T_{c} \simeq 180 \mathrm{MeV}$. In both cases it thus assumed that

$$
\Pi_{L}=\Pi_{T} /\left(1-\mu^{2}\right)=m_{p l}
$$

in (B1) which leads to

$$
Q\left(\frac{q_{\max }}{q_{\min }}\right)=\frac{1}{2}\left[\ln \left(1+\frac{q_{\max }^{2}}{m_{p l}^{2}}\right)-\frac{q_{\max }^{2}}{m_{p l}^{2}+q_{\max }^{2}}\right] .
$$


With $q_{\text {max }}^{(g g)}=3.0 T, q_{\text {max }}^{(g q)}=3.8 T, q_{\text {max }}^{(q q)}=4.8 T$ and $m_{p l}=$ $1.1 T$ we find $Q\left(q_{\max }^{(g g)} / q_{\min }\right)=0.626, Q\left(q_{\max }^{(g q)} / q_{\min }\right)=$ 0.819 and $Q\left(q_{\max }^{(q q)} / q_{\min }\right)=1.024$. These values enter the $\alpha_{s} \gtrsim 0.1$ expressions of Eqs. (38) and (41).
[1] S. Gavin, Nucl. Phys. B435, 826 (1984).

[2] A. Hosoya and K. Kajantie, Nucl. Phys. B250, 666 (198

[3] P. Danielewicz, Phys. Lett. B146, 168 (1984).

[4] G. Baym, H. Monien, C. J. Pethick, and D. G. Ravenh Phys. Rev. Lett. 64, 1867 (1990); G. Baym, H. Monien a C. J. Pethick, Proc. XVI Int. Workshop on Gross Prop ties of Nuclei and Nuclear Excitations, Hirschegg, (ed. Feldmeier, GSI and Institut für Kernphysik, Darmsta 1988), p. 128; and C.J. Pethick, G. Baym, and H. Moni Nucl. Phys. A498, 313c (1989).

[5] G. Baym, H. Monien, C. J. Pethick, and D. G. Ravenh Nucl. Phys. A525, 415c (1991); G. Baym, H. Heiselbe H. Monien, C. J. Pethick, and J. Popp, Nucl. Phys. A 5 569c (1992).

[6] H. Heiselberg and C.J. Pethick, Phys. Rev. D48, 29 (1993); H. Heiselberg, G. Baym, and C. J. Pethick, Nu Phys. B (Proc. Suppl.) 24B, 144 (1991);

[7] B. L. Combridge, J. Kripfganz, J. Ranft, Phys. Lett. 70 234 (1977).

[8] H. A. Weldon, Phys. Rev. D26, 1394 (1982).

[9] M. B. Kislinger and P. D. Morley, Phys. Rep. 51, 63 (197 O. K. Kalashnikov, Fortschr. Phys. 32, 525 (1984).

[10] G. Baym and C. J. Pethick, Landau Fermi-liquid theo concepts and applications, (J. Wiley and Sons, New Yo 1991).

[11] M. H. Thoma, Phys. Lett. B269, 144 (1991); and in R 20 .

[12] M. Gao, Phys. Rev. D41, 626 (1990); A. Irback et Nucl. Phys. B363, 34 (1991).

[13] L. Xiang and E. Shuryak, SUNY-NTG-93-24.

[14] O. K. Kalashnikov, Phys. Lett. B 279, 367 (1992).

[15] E. Braaten, R. D. Pisarski, Phys. Rev. D42, 2156 (199

[16] H. Heiselberg and C. J. Pethick, Phys. Rev. D47, R7 (1993); C. P. Burgess and A. L. Marini, Phys. Rev. D R17 (1992); A. Rebhan, Phys. Rev. D48, 482 (1992).

[17] A. D. Linde, Phys. Lett. B96, 289 (1980).

[18] E. M. Lifshitz and L. P. Pitaevskii, Physical Kinet (Pergamon, New York, 1981), §61.

[19] A. Selikhov and M. Gyulassy, Phys. Lett. B316, (1993); CU-TP-610/93 and in 20; H. Heiselberg, in R 20 .

[20] U. Heinz, proc. of LBL workshop on "Pre-equilibri Parton Dynamics in Heavy Ion Collisions", august september 3, 1993. 
FIG. 1. Feynman diagram for gluon-gluon scattering in the t-channel. The lines in the loops can be either quark or gluon propagators.

FIG. 2. The collision geometry. For small momentum transfer, $q \ll p_{1}, p_{2}$, energy and momentum conservation requires $\cos \theta_{1}=\cos \theta_{2}=\omega / q$.

FIG. 3. The gluon viscosity for $N_{f}=3$ assuming Debye and dynamical screening (dashed curve), a constant screening mass $m_{p l}=1.1 T$ (dashed-dotted curve) and the minimal one (full curve).

FIG. 4. The quark, gluon and total viscosities for $N_{f}=3$.

FIG. 5. The quark, gluon and total viscosities for $N_{f}=2$.

FIG. 6. The function $f / x^{2}$ as determined by (A15). Also shown are the limits of (A16), (A17) and the simple ansatz $f=x^{2}$. 
This figure "fig1-1.png" is available in "png" format from: http://arxiv.org/ps/hep-ph/9401309v1 
This figure "fig2-1.png" is available in "png" format from: http://arxiv.org/ps/hep-ph/9401309v1 
This figure "fig1-2.png" is available in "png" format from: http://arxiv.org/ps/hep-ph/9401309v1 
This figure "fig2-2.png" is available in "png" format from: http://arxiv.org/ps/hep-ph/9401309v1 
This figure "fig1-3.png" is available in "png" format from: http://arxiv.org/ps/hep-ph/9401309v1 
This figure "fig2-3.png" is available in "png" format from: http://arxiv.org/ps/hep-ph/9401309v1 